\title{
CLOSED CURVES OF CONSTANT TORSION. III
}

\author{
JOEL L. WEINER
}

\begin{abstract}
In this note we show that there exist closed regular $C^{3}$ space curves $\alpha$ with curvature $\kappa>0$ and nonzero constant torsion $\tau$ whose total torsion $\int_{\alpha} \tau d s$ is arbitrarily small. In so doing, we give another proof of the existence of closed curves of nonzero constant torsion. This note shows that Conjecture 2 in [2] is incorrect since the preceding statement is equivalent to the statement that there exist closed curves of constant torsion $\tau=1$ whose length is arbitrarily small.
\end{abstract}

Let $\alpha: S^{1} \rightarrow \mathbf{E}^{3}$ be a closed $C^{1}$ space curve. The Peano direction of $\alpha$ is the vector $P_{\alpha}$ defined by

$$
P_{\alpha}=\int_{S^{1}} \alpha \times d \alpha
$$

where $X$ is the usual cross-product in space. The Peano direction of $\alpha$ has two noteworthy properties:

(1) $P_{\alpha}$ is independent of the Cartesian coordinate system on $\mathbf{E}^{3}$ and, in particular, the origin.

(2) If $\alpha_{z}$ is the projection of $\alpha$ onto a plane perpendicular to the vector $z(\|z\|=1)$, then

$$
P_{\alpha_{z}}=\int_{S^{1}} \alpha_{z} \times d \alpha_{z}
$$

equals the projection of $P_{\alpha}$ onto $z ;\left(P_{\alpha} \cdot z\right) z$.

Let $O$ be the origin of $\mathbf{E}^{3}$ with respect to a Cartesian coordinate system on $\mathbf{E}^{3}$ and let $S^{2}$ be the unit sphere centered at $O$. Let $\alpha: S^{1} \rightarrow \mathbf{E}^{3}$ be a regular $C^{2}$ space curve with positive curvature. We may define $\alpha$ 's binormal indicatrix, $\beta: S^{1} \rightarrow S^{2}$, by regarding the binormal vector field along $\alpha$ as a curve on $S^{2}$. In [2] we give conditions for the existence of a closed regular $C^{3}$ space curve $\alpha$ with positive curvature and nonzero constant torsion in terms of $\alpha$ 's tangent indicatrix $\sigma$. We now restate those conditions in terms of $\beta$.

Proposition. There exists a closed regular $C^{3}$ space curve with curvature $\kappa>0$ and torsion $\tau=1$ if and only if there exists a closed curve $\beta$ on $S^{2}$

Received by the editors October 20, 1976.

AMS (MOS) subject classifications (1970). Primary 53A05.

Key words and phrases. Closed space curves, constant torsion, total torsion, binormal indicatrix, Peano direction.

1 Supported in part by NSF Grant No. MCS 76-08320. 
satisfying the following properties:

(A) $\beta$ is a regular $C^{2}$ curve with positive geodesic curvature.

(B) $P_{\beta}=0$.

Proof. The Proposition follows immediately from the Proposition of [2] and the fact that the tangent indicatrix and binormal indicatrix are polars of each other under the spherical polarity; see [1] for the pertinent facts about the spherical polarity.

The curve $\beta$ on $S^{2}$ in the preceding Proposition turns out to be the binormal indicatrix of the space curve $\alpha$ of constant torsion $\tau=1$. Hence the length of $\beta$ is the total torsion of the space curve $\alpha$. We will establish the existence of closed curves of nonzero constant torsion whose total torsion is arbitrarily small by constructing closed curves on $S^{2}$ which satisfy properties (A) and (B) and whose length is arbitrarily small.

Let $z$ be a unit vector, $Z$ a line through $O$ in the direction of $z$, and $\pi$ a plane through $O$ perpendicular to $z$. Let $p: S^{2} \rightarrow \pi$ be projection in the $z$ direction from $S^{2}$ onto $\pi$; note that $p$ sends any curve $\beta$ on $S^{2}$ into $\beta_{z}$.

If we construct a curve $\beta$ on $S^{2}$ which is invariant under rotations of $2 \pi / 3$ radians about $Z$ and for which $P_{\beta_{z}}=0$, then $P_{\beta}=0$ by symmetry and property (2) of the Peano direction. But note that $\beta$ is invariant under rotations of $2 \pi / 3$ radians about $Z$ if $\beta_{z}$ lies in the open unit disk $D$ about $O$ in $\pi$ and is invariant under such rotations. Hence we need only construct a plane curve $\beta_{z}$ in $D$ which is invariant under rotations of $2 \pi / 3$ radians about $Z$, or $O$, and for which $P_{\beta_{z}}=0$ in order to get a curve $\beta$ on $S^{2}$ for which $P_{\beta}=0$. Simply let $\beta=p^{-1} \circ \beta_{z}$ where $p^{-1}$ maps $D$ onto a suitably chosen open hemisphere $H$ of $S^{2}$.

Therefore let $\beta_{z}$ be a closed regular $C^{2}$ curve in $D$ satisfying the properties:

(a) $\beta_{z}$ has positive curvature.

(b) $\beta_{z}$ is invariant under rotations of $2 \pi / 3$ radians about $O$ and $P_{\beta_{z}}=0$.

Requiring that $\beta_{z}$ have positive curvature does not, of course, imply that $\beta=p^{-1} \circ \beta_{z}$ on $S^{2}$ has positive geodesic curvature. However, $p: H \rightarrow D$ is a coordinate system on $H$ which is almost normal at $H \cap Z$, i.e., the metric tensor $g_{i j}=\delta_{i j}$ and the Christoffel symbols $\Gamma_{j k}^{i}=0$ for this coordinate system at $H \cap Z$. For $0<r<1, r \beta_{z}$ still has properties (a) and (b); in fact, the curvature of $r \beta_{z} \rightarrow \infty$ as $r \rightarrow 0$. Moreover, as $r \rightarrow 0, r \beta_{z}$ lies in smaller and smaller neighborhoods of $O$ on which $p^{-1}$ changes the geodesic curvature by lesser and lesser amounts. Hence for sufficiently small $r, p^{-1} \circ\left(r \beta_{z}\right)$ satisfies (A) and (B), provided $H$ is chosen to be the hemisphere for which $p^{-1} \circ\left(r \beta_{z}\right)$ has positive geodesic curvature. Finally, note that the length of $p^{-1} \circ\left(r \beta_{z}\right) \rightarrow$ 0 as $r \rightarrow 0$.

It is easy to construct examples of closed regular $C^{2}$ plane curves lying in an open unit disk about $O$ satisfying (a) and (b). For example, $\beta_{z}: \mathbf{R} \rightarrow \mathbf{E}^{2}$ given by

$$
\beta_{z}(\theta)=\left(\frac{1}{2} \cos \theta+(\sqrt{2} / 4) \cos 2 \theta, \frac{1}{2} \sin \theta-(\sqrt{2} / 4) \sin 2 \theta\right)
$$


induces the required curve $\beta_{z}: S^{1} \rightarrow \mathbf{E}^{2}$.

In fact for the given $\beta_{z}$ we computed $\beta=p^{-1} \circ \beta_{z}$, and the numerically integrated $\beta \times d \beta / d \theta$ to obtain a closed curve of constant torsion. The projection of the resulting curve on the $x, y$ and $x, z$ planes is shown in Figures 1 and 2 , respectively.

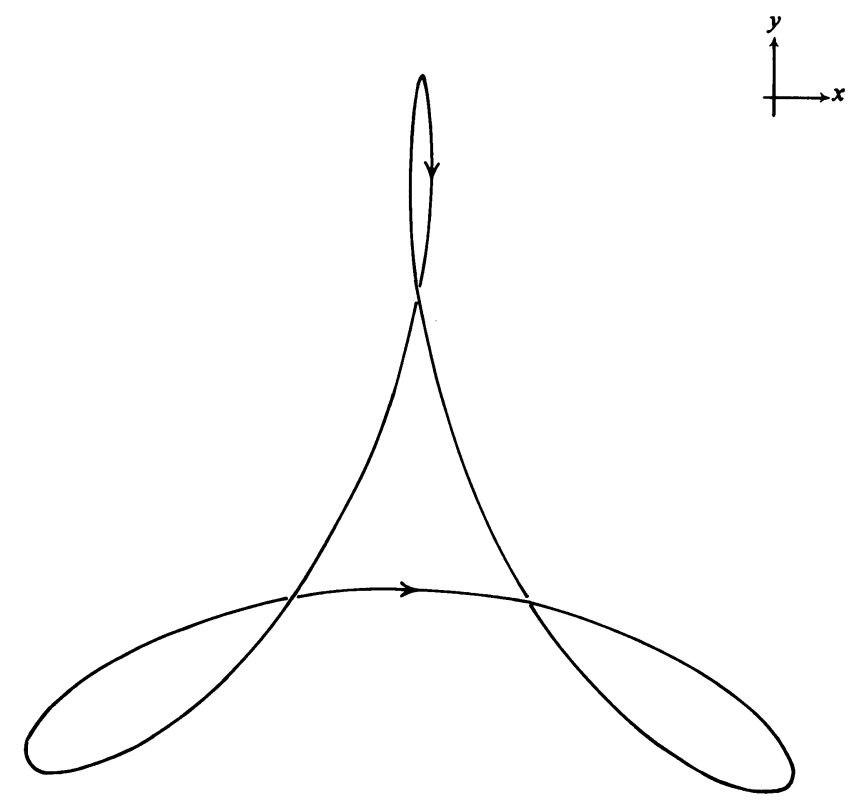

Figure 1

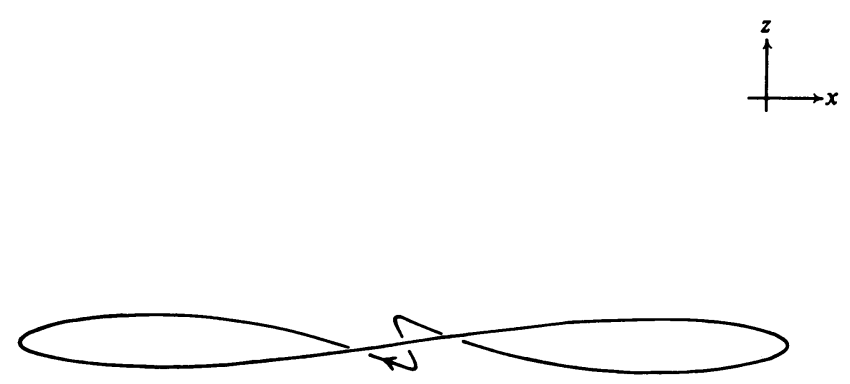

FIGURE 2

\section{REFERENCES}

1. Werner Fenchel, On the differential geometry of closed space curves, Bull. Amer. Math. Soc. 57 (1951), 44-54. MR 12, 634.

2. Joel L. Weiner, Closed curves of constant torsion, Arch. Math. (Basel) 25 (1974), 313-317. MR 49 \#11437.

Department of Mathematics, University of Hawail, Honolulu, Hawail 96822 